\title{
SACRAMENTO PEAK MAGNETOGRAPH
}

\author{
RICHARD B. DUNN \\ Sacramento Peak Observatory, Air Force Cambridge Research Laboratories, \\ Sunspot, New Mexico, U.S.A.
}

\begin{abstract}
The Sac Peak magnetograph (DZA) has been modified from Evans' original scheme so that it measures the displacement of the right and left hand circularly polarized lines separately. The computer reduction calculates the Zeeman and radial velocity signals. A grating servo system has been added to correct for slow temperature drifts in the spectrograph. A paper-tape reader controls the raster scan and the formatting of data on to magnetic tape.
\end{abstract}

The Sacramento Peak Magnetograph has been briefly described by Evans (1966) in his summary of Solar Magnetographs. He refers to this device as the DZA, which is an abbreviation for Doppler-Zeeman Analyzer. The DZA contains servos that continually re-center the spectral lines on analyzing slits of fixed separation. When operating on strongly split lines, it overcomes the saturation problems of a Babcock magnetograph. The DZA operates as a longitudinal magnetograph in the presence of both $\sigma$ and $\pi$ components unless the components are completely separated, in which case it measures total field.

This paper describes a number of significant modifications to the instrument and COMPACT SPECTROGRAPH

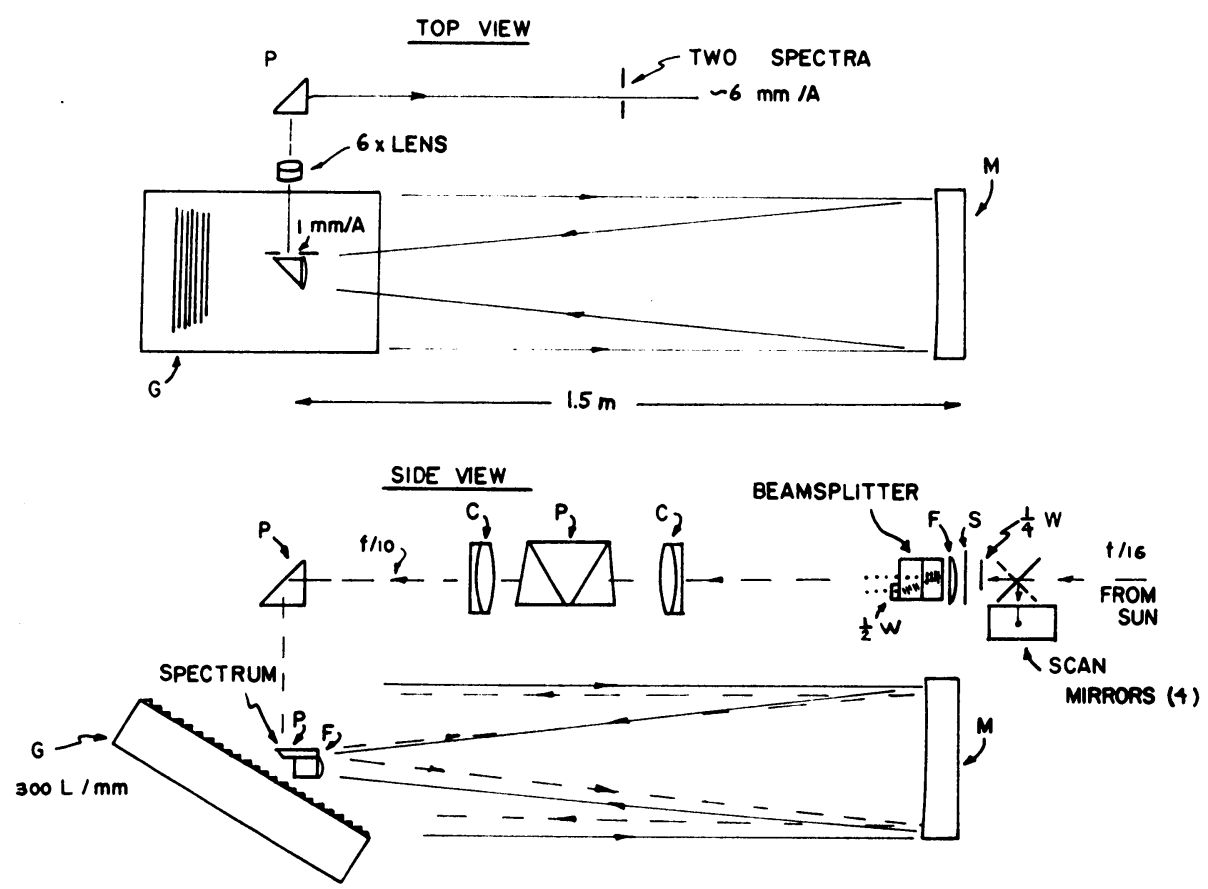

Fig. 1. Compact spectrograph. 
the addition of a data collection system. Utilizing a compact spectrograph designed by himself, and shown schematically in Figure 1, Evans formed two spectra simultaneously, one above the other. The top one is analyzed for the right-handed circularly polarized component, the lower for the left-hand component. The analyzer consists of a $\frac{1}{4}$ wave plate in front of the spectrograph slit and a polarizing beamsplitter immediately after the slit. The beamsplitter is made from two pieces of cleaved calcite with a $\frac{1}{2}$ wave plate in between to equalize the optical path length between the two images. Another $\frac{1}{2}$ wave plate cemented to one-half of the exit face of the beamsplitter rotates the plane of polarization of one of the beams so that it coincides with the other and so that they both coincide with the azimuth of highest reflectance of the grating.

There is no chopping between the two circularly polarized components because there is no KD*P crystal in the analyzer. The DZA simply measures the displacement of the spectral line relative to its average position by balancing the light passing through a slit on each side of the line as shown in Figure 2. An unbalance causes an error signal
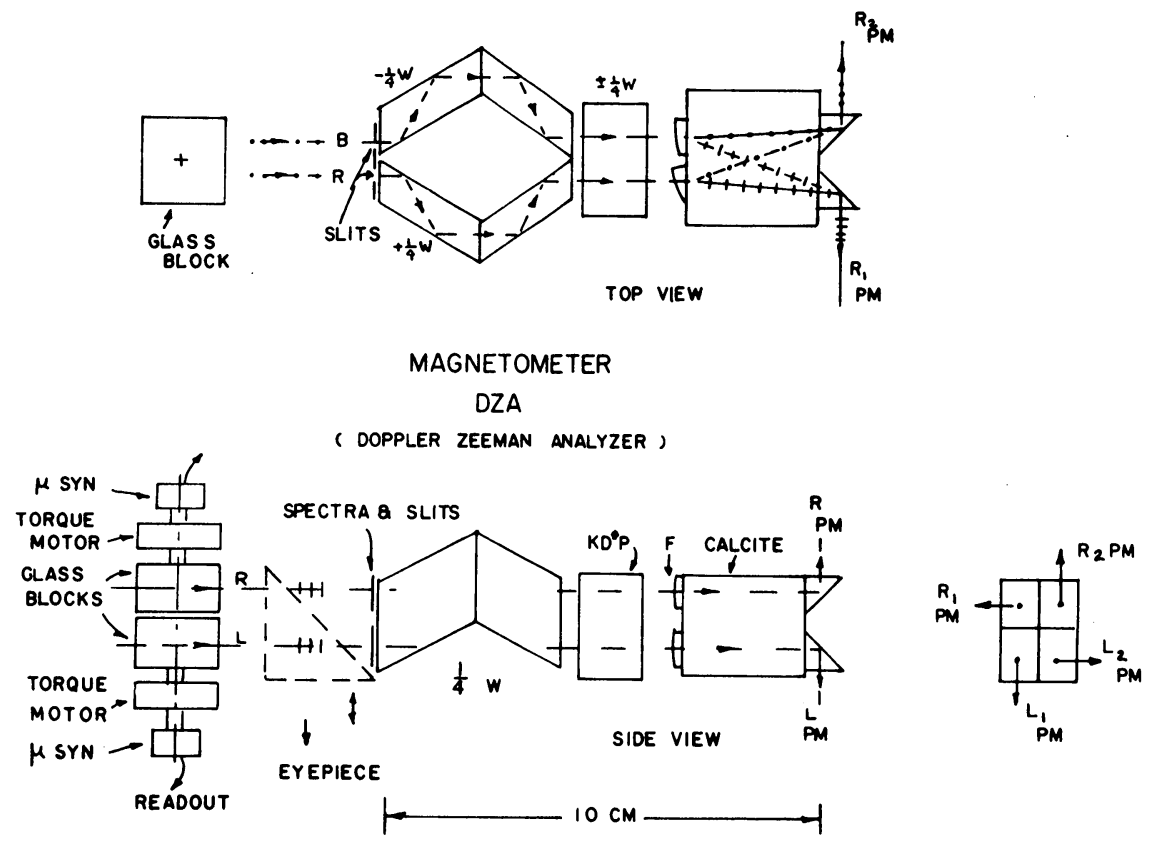

Fig. 2. Sacramento Peak magnetograph.

that is amplified in a servo loop to drive a torque motor that, in turn, rotates a cube of glass until the signal is nulled. The amount of rotation of the cube, and thus the displacement of the line, is measured by a small transformer with a rotating armature called a 'Microsyn'.

The $\frac{1}{4}$ wave plate, $\mathrm{KD}^{*} \mathrm{P}$ crystal and calcite polarizer are simply a convenient means devised by Evans for chopping between the two slits to develop the error signal for the servo system. The two spectra are already polarized by the analyzer at the slit 
of the spectrograph. The chopping is accomplished by adding a fixed plus quarter wave to one slit and a minus quarter wave to the other. Evans used achromatic wave plates consisting of Fresnel rhombs. During one portion of the chop the KD*P adds a plus $\frac{1}{4}$ wave to the light to linearly polarize it again. The subsequent calcite beamsplitter sends the light from the red slit to the $R_{1}$ photomultiplier and the light from the blue slit to the $R_{2}$ photomultiplier. On the other portion of the chop the KD*P crystal adds a minus $\frac{1}{4}$ wave and the light from the blue slit is sent to the $R_{1}$ photomultiplier and that from the red slit is sent to the $R_{2}$ photomultiplier. All the light is utilized in this arrangement. The beamsplitters are made from calcite and the surfaces are coated so that the loss of light due to absorption is small. The signals from $R_{1}$ and $R_{2}$ are added to form the $R$ error signal. An identical arrangement is used in the $L$ channel.

The original DZA utilized two glass cubes in series so that the Microsyns measured line displacements proportional to Zeeman and Doppler signals instead of to $R$ and $L$. In adapting the DZA to digital recording we decided to eliminate this feature in order to allow corrections to be made by the computer to the individual $R$ and $L$

\section{MAGNETOGRAPH ELECTRONICS}

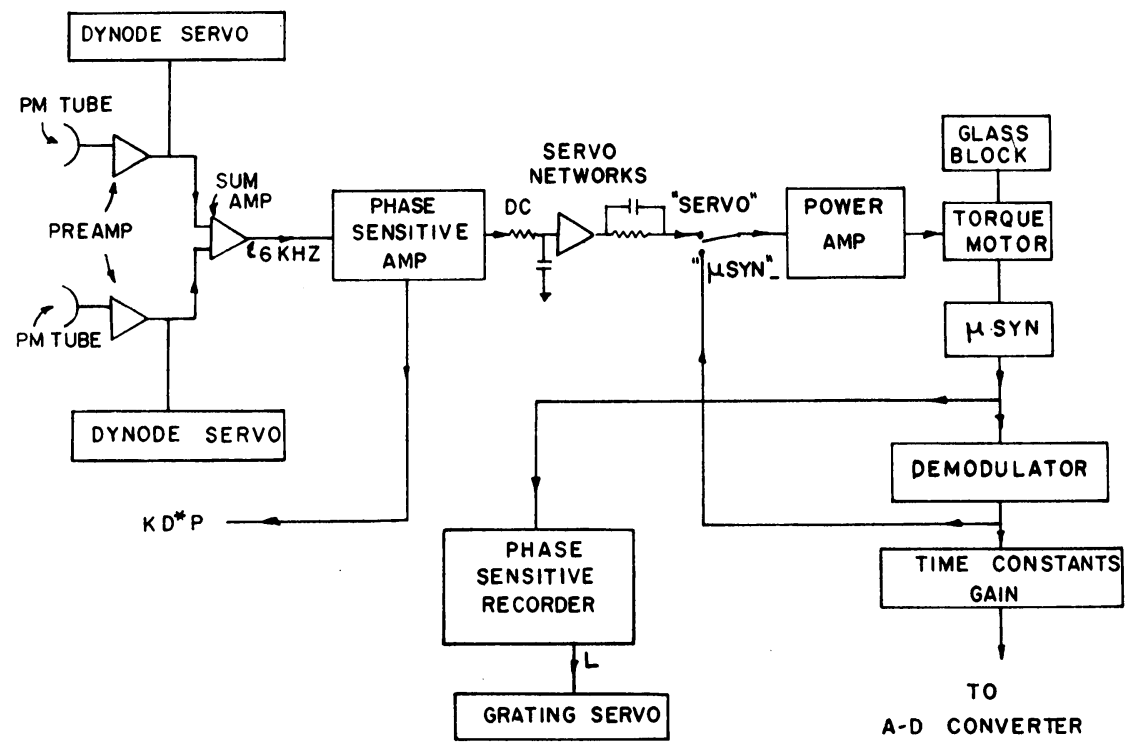

Fig. 3. Magnetograph electronics.

channels. The computer then adds and substracts the $R$ and $L$ Microsyn signals to form the Doppler and Zeeman signals.

The block diagram for the electronics is shown in Figure 3. The output of the photomultiplier preamps is summed in an operational amplifier before it is phasedetected by a phase-sensitive amplifier. The output of the phase-sensitive amplifier is 
compensated to correct the frequency response of the servo system and drives the torque motor that rotates the glass block. The output of the Microsyn is demodulated and sent to a digital tape system consisting of a multiplexer, an analog-to-digital converter, a tape format controller and a 7-track tape drive. An auxiliary analog recorder traces the $R$ and $L$ signals and by means of a circuit that adds and subtracts the $R$ and $L$ signals, traces the Zeeman and Doppler signals.

The dynodes of the photomultiplier tubes are servoed to hold the DC output of each photomultiplier constant so that the servos operate at constant gain even while measuring the fields of sunspots.

Two of the four dynode signals, the coordinates on the Sun, and the $R$ and $L$ signals are multiplexed and recorded on the magnetic tape.

The format of the scan is predetermined by a paper tape and machine-tool controller that programs Right Ascension and Declination stepping motors to drive a scanner in front of the slit. One step corresponds to $0.3 \mathrm{~s}$ of arc. Auxiliary control characters on the paper tape cause the clock, record and end-of-file gaps, and the information set manually on digital switches to be recorded on the magnetic tape.

In order to compensate for thermal drifts, an additional servo very slowly rotates the grating to keep the spectral lines centered on the DZA. It derives its signal from the recorder output.

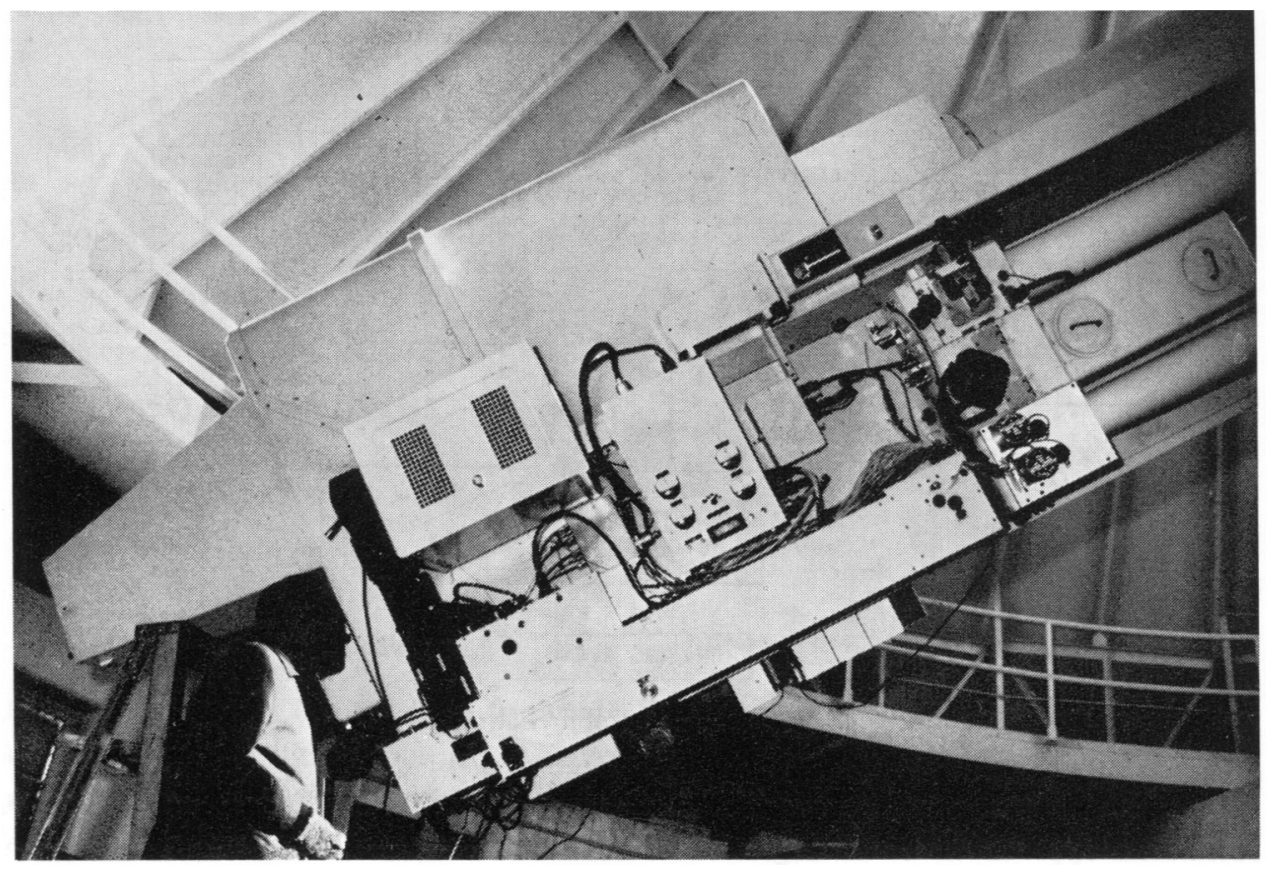

Fig. 4. Photograph of magnetograph. The DZA is on top of the compact spectrograph. Light comes into the system from the right hand side. The scanner is immediately to the right of the compact spectrograph. A small rack mounted above the DZA contains the power supplies for the stepping motors. To the right is a second box of electronics containing the dynode servo systems. 
The system has been used to record data for several thousand scan series. The taperecorded records are analyzed in a digital computer. Currently it is possible to produce three (3) contoured maps - of magnetic field, velocity field and brightness - in about two minutes. We record the maps on microfilm or on drawing paper for detailed analysis. Because the instrument measures only relative displacements of the $L$ and $R$ components of the line, a substantial portion of the reduction process involves the location and removal of the relative zeros of magnetic and velocity field. Magnetic field measurements have been restricted mainly to active regions, since it is possible, in that case, to assume that the regions of spatially smooth field surrounding the steep and irregular fields of active centers are regions of approximately zero field. That is, we find the zero by fitting the flat field regions - not by equating mean measured field with zero field.

The DZA is still undergoing changes. At present, we are adding tachometer feedbacks to the torque motor servos to improve the low frequency response and we are changing the 1P21 photomultiplier tubes to an extended red-sensitive tube. We are in the final stages of installation of a data-link between the DZA and our medium-size XDS computer. We want to send the data directly to the computer during observations because data can be recorded more rapidly that way than with

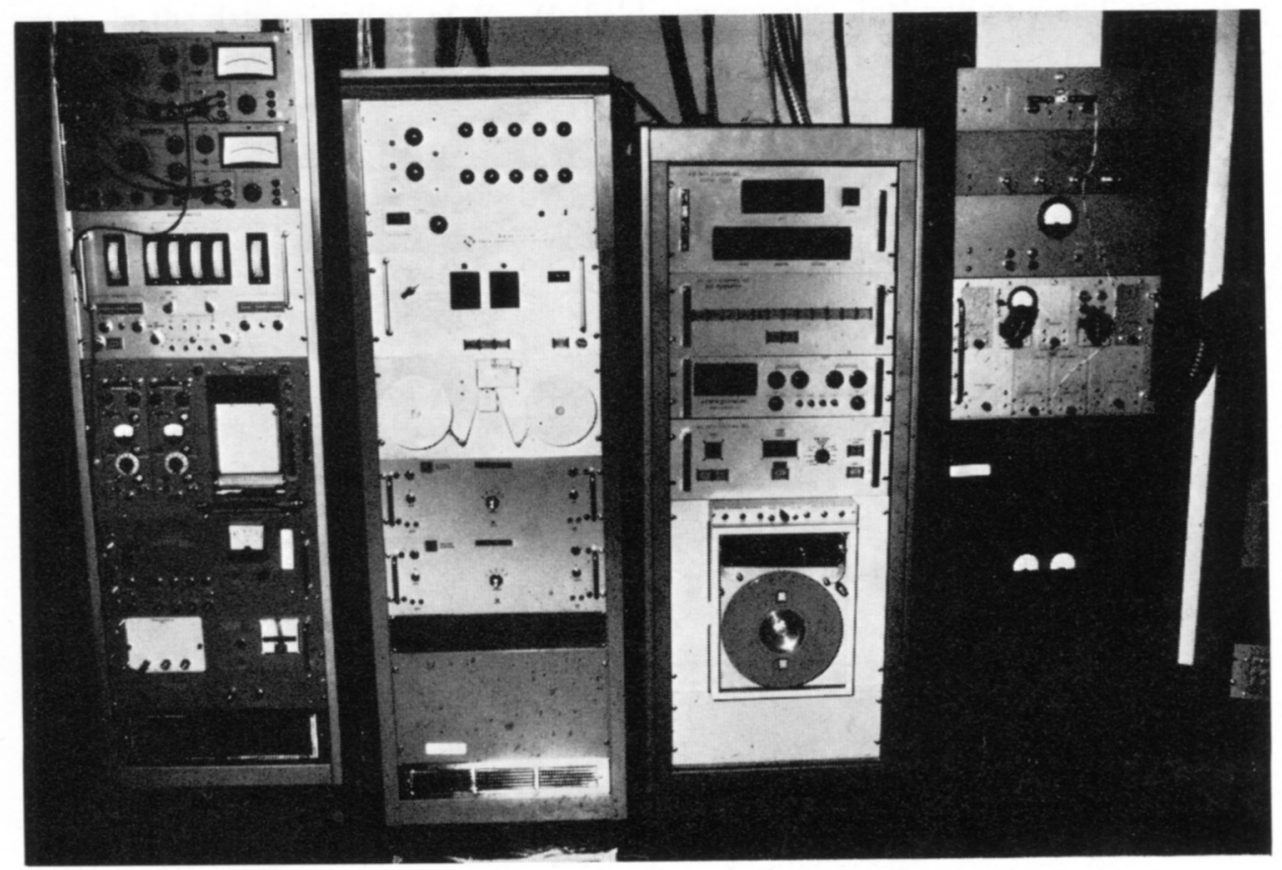

Fig. 5. Photograph of magnetograph electronics and data collection system. The phase sensitive amplifiers are in the left rack together with servo control electronics. The center rack contains the machine-control system with the paper-tape reader and servo power amplifiers. The third rack contains the digital data collection system including the clock, multiplexer, analog-to-digital converter, controller, and magnetic tape unit. 
the currently-used incremental tape recorder. Also, we will be able to produce nearly real-time maps of the velocity, magnetic and brightness fields being observed. The maps will be drawn on a storage-type oscilloscope and transmitted via television to the observer at the telescope. We feel that this feedback to the observer will significantly improve the quality of the data obtained.

Photographs of the instrument and its electronics are shown in Figure 4 and 5. The DZA presently operates with a $40 \mathrm{~cm}$ telescope. Maps made from the instrument will be shown in other papers presented by Sac Peak staff at this meeting.

\section{Reference}

Evans, J. W.: 1966, Atti Del Convegno Sui Campi Magnetici Solari, (ed. by M. Cimino), G. Barbèra, Firenze. 